\title{
On-Chip Maxwell's Demon as an Information-Powered Refrigerator
}

\author{
J. V. Koski, ${ }^{1}$ A. Kutvonen, ${ }^{2}$ I. M. Khaymovich, ${ }^{1,3}$ T. Ala-Nissila, ${ }^{2,4}$ and J. P. Pekola ${ }^{1}$ \\ ${ }^{1}$ Low Temperature Laboratory, Department of Applied Physics, \\ Aalto University School of Science, P.O. Box 13500, FI-00076 Aalto, Espoo, Finland \\ ${ }^{2}$ COMP Center of Excellence, Department of Applied Physics, \\ Aalto University School of Science, P.O. Box 11000, FI-00076 Aalto, Espoo, Finland \\ ${ }^{3}$ Institute for Physics of Microstructures, Russian Academy of Sciences, 603950 Nizhni Novgorod, GSP-105, Russia \\ ${ }^{4}$ Department of Physics, Brown University, Providence Rhode Island 02912-1843, USA \\ (Received 1 October 2015; revised manuscript received 4 November 2015; published 30 December 2015)
}

\begin{abstract}
We present an experimental realization of an autonomous Maxwell's demon, which extracts microscopic information from a system and reduces its entropy by applying feedback. It is based on two capacitively coupled single-electron devices, both integrated on the same electronic circuit. This setup allows a detailed analysis of the thermodynamics of both the demon and the system as well as their mutual information exchange. The operation of the demon is directly observed as a temperature drop in the system. We also observe a simultaneous temperature rise in the demon arising from the thermodynamic cost of generating the mutual information.
\end{abstract}

DOI: 10.1103/PhysRevLett.115.260602

PACS numbers: 05.70.-a, 65.40.gd, 72.15.Eb, 73.23.Hk

Thermodynamic processes are governed by fundamental laws, of which the first, conservation of energy, is paramount in all fields of physics and cannot be violated at any level of description known to date. The second law in turn states that entropy, the measure of disorder, of a closed system cannot decrease. This has most important consequences, such that heat flows from hot to cold, irreversible processes must dissipate work, and devices of perpetual motion are impossible. To challenge this law, Maxwell presented a thought experiment in 1867 of a "finite being" capable of accurately measuring the velocity of molecules [1]. It would act between two separated reservoirs, permitting only fast molecules to enter one reservoir, while allowing only the slow ones to the other. Under such a process heat is transferred from cold to hot, apparently in violation of the second law. This idea, coined as "Maxwell's demon" by Lord Kelvin, has over a century spurred further research on the relation between information and energy establishing quantitative relations [2-12]. Ongoing progress in nanotechnology has also provided concrete means to test such relations experimentally [13-23], thus reigniting acute interest in actually constructing a demon.

Recently, several theoretical proposals on configurations including both the system as well as the demon have been presented [7,24-26]. Such a configuration is known as an autonomous Maxwell's demon, for the fact that the measurement and feedback operation takes place internally. Here, we experimentally realize an all-in-one Maxwell's demon, whose operation principle is illustrated in Fig. 1(a). The system is a single-electron transistor (SET) [27], formed by a small normal metallic island connected to two normal metallic leads by tunnel junctions. The two junctions permit electron transport by tunneling, and are assumed to be identical (both with the same resistance $R_{s}$ ). The demon measures the number of electrons on the system island, and applies feedback as depicted in Fig. 1(a). When an electron tunnels to the island, the demon traps it with a positive charge (panels 1 and 2). Conversely, when an electron leaves the island, the demon applies a negative charge to repel further electrons that would enter the island (panels 3 and 4). The system electrodes contain a reservoir of conduction electrons whose thermal excitations provide sufficiently high energy carriers to overcome the trapping or repulsion induced by the demon, contributing heat $Q=-\Delta E$, where $\Delta E$ is the energy cost of the tunneling event. In doing so, the system entropy decreases as $\Delta S_{s}=Q / T_{s}$, where $T_{s}$ is the system reservoir temperature; i.e., the demon extracts information of tunneling electrons to apply feedback that causes the entropy of the system to decrease. While the configuration resembles theoretical proposals on quantum dots $[25,28,29]$, and shares features with the Coulomb drag effect [30,31], it constitutes a genuine autonomous Maxwell's demon where only information, not heat, is directly exchanged between the system and the demon.

Our experimental, autonomous realization of the cycle in Fig. 1(a) relies on coupling the system island capacitively to a single-electron box, a small normal metallic island connected by a tunnel junction with resistance $R_{d}$ to a single normal metallic lead. Here, the single-electron box undertakes the role of the demon. The resulting Hamiltonian is

$$
\begin{aligned}
H(n, N)= & E_{s}\left(n-n_{g}\right)^{2}+E_{d}\left(N-N_{g}\right)^{2} \\
& +2 J\left(n-n_{g}\right)\left(N-N_{g}\right),
\end{aligned}
$$


(a)

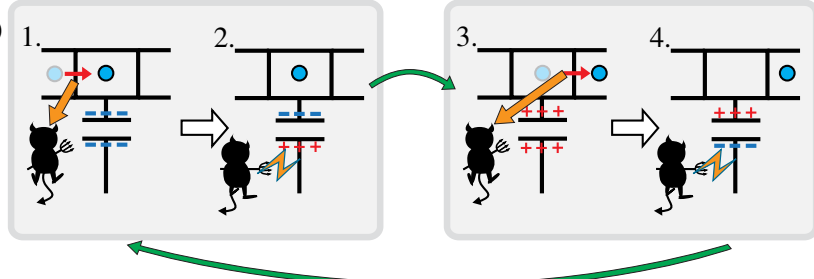

(b)

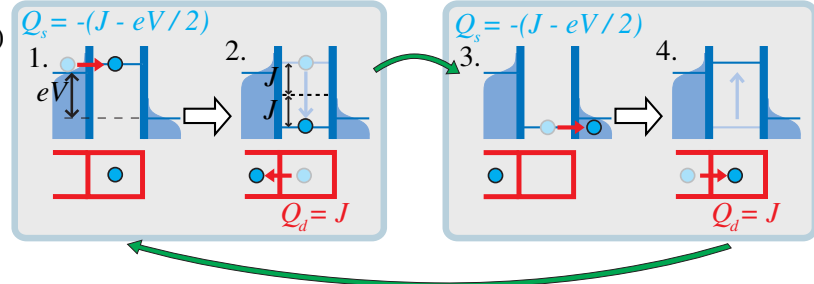

FIG. 1 (color online). Operation principle. (a) The demon monitors the system (a single-electron transistor) for electrons that tunnel into (panel 1) or out of (panel 3) the island. It then immediately performs a feedback by applying a positive charge to trap (panel 2) or negative charge to repel (panel 4) the electrons. Coulomb blockade ensures that only either one or zero electrons reside in the system island. The electrons are always tunneling against the potential induced by the demon, and therefore the system cools down. (b) Energetics of the system under voltage bias $V$ in the experimental and autonomous realization of the cycle in (a) with another single-electron structure operating as the demon. The conduction electrons of the system follow Fermi distribution, providing electrons that can overcome the energy cost $J-e V / 2$, where $J$ is the coupling energy between the system and the demon; however, in doing so, the system cools down by an equal amount. The energy $J$ is dissipated by the demon as it reacts, changing the projected energy cost experienced by the electron tunneling in the system from $-\mathrm{J}-\mathrm{eV} / 2$ to $J-e V / 2$. Note that here the system island is drawn without Fermi distribution for simplicity. Also, the described operation could, in principle, be performed nonautonomously by externally measuring the system state and changing the energy of the system as feedback; see, e.g., Ref. [25].

where the dynamic variables $n$ and $N$ are the net number of electrons that have entered the system and the demon islands, respectively. $E_{s}$ and $E_{d}$ are their charging energies, while $J>0$ describes their mutual Coulomb interaction and is essential for the device operation. The state $(n, N)$ evolves when an electron tunnels through a junction. $n$ changes to $n_{\overline{(+)}} 1$ when an electron tunnels from (to) the system island. Correspondingly, $N$ changes to $N_{(\overline{+})} 1$ when it transfers from (to) the demon island. Constant external control parameters $n_{g}$ and $N_{g}$ govern the system current and the coupling of the demon to the system, respectively. The system is voltage $(V)$ biased, such that the electron (with elementary charge $-e$ ) tunneling in the direction of (against) the voltage bias experiences an energy cost $\Delta E=\Delta H_{(+)} e V / 2$, where $\Delta H=H(n \pm 1, N)-H(n, N)$ for changing $n$ is given by Eq. (1). Similarly, for the electron tunneling in the demon, $\Delta E=H(n, N \pm 1)-H(n, N)$.

The interaction between the system and the demon is maximized by setting $n_{g}=N_{g}=0.5$, producing the
Hamiltonian $H(n, N)=J(2 n-1)(2 N-1) / 2$ and energetics depicted in Fig. 1(b). We furthermore require $\mathrm{eV}$, $k_{B} T \ll E_{s}, E_{d}$, such that only the lowest energy states of Eq. (1) are available, where both $n$ and $N$ are practically limited to two possible values, 0 and 1 . States $(n=0$, $N=1)$ and $(n=1, N=0)$ are charge neutral, both with energy $-J / 2$. Here, we refer to either of the states as "ground" or $g$. The state $(n=0, N=0)$ has an overall positive charge and $(n=1, N=1)$ an overall negative charge. We refer to them as "charged" or $c$, both with energy $J / 2$. Any single tunneling event will take $g$ to $c$ or $c$ to $g$, with respective $\Delta H_{g \rightarrow c}=J=-\Delta H_{c \rightarrow g}$. We assume that the system is at uniform temperature $T_{s}$ while the temperature of the demon is $T_{d}$, such that the occupation probability distribution $P_{n, N}$ obeys $P_{0,1}=P_{1,0} \equiv P_{g} / 2$ and $P_{0,0}=P_{1,1} \equiv P_{c} / 2$, with $P_{g}=\Gamma_{c \rightarrow g} /\left(\Gamma_{g \rightarrow c}+\Gamma_{c \rightarrow g}\right)$ and $P_{c}=\Gamma_{g \rightarrow c} /\left(\Gamma_{g \rightarrow c}+\Gamma_{c \rightarrow g}\right)$. Here, with notation $J_{ \pm} \equiv$ $J \pm \mathrm{eV} / 2$, the term $\Gamma_{c \rightarrow g}=\Gamma_{s}\left(-J_{+}\right)+\Gamma_{s}\left(-J_{-}\right)+\Gamma_{d}(-J)$ is the overall transition rate from $c$ to $g$, while $\Gamma_{g \rightarrow c}=$ $\Gamma_{s}\left(J_{-}\right)+\Gamma_{s}\left(J_{+}\right)+\Gamma_{d}(J)$ is the corresponding overall transition rate from $g$ to $c$ as a sum of rates in the system in the direction of bias, against the bias, and the transition rate in the demon, respectively. The transition rates are

$$
\Gamma_{s / d}(\Delta E)=\frac{1}{e^{2} R_{s / d}} \frac{\Delta E}{e^{\Delta E / k_{B} T_{s / d}}-1} .
$$

The charge current in the system is $I=(e / 2)\left[\Gamma_{s}\left(J_{-}\right)-\right.$ $\left.\Gamma_{s}\left(J_{+}\right)\right] P_{g}+(e / 2)\left[\Gamma_{s}\left(-J_{+}\right)-\Gamma_{s}\left(-J_{-}\right)\right] P_{c}$ and the total heat generation rate there is

$$
\begin{aligned}
\dot{Q}_{s}= & -\left[J_{-} \Gamma_{s}\left(J_{-}\right)+J_{+} \Gamma_{s}\left(J_{+}\right)\right] P_{g} \\
& +\left[J_{-} \Gamma_{s}\left(-J_{-}\right)+J_{+} \Gamma_{s}\left(-J_{+}\right)\right] P_{c},
\end{aligned}
$$

reflecting the fact that if the demon successfully maintains a high $P_{g}$ by feedback, as in Fig. 1(b), $\dot{Q}_{s}$ is negative. Similarly, the rate of heat generation in the demon is

$$
\dot{Q}_{d}=-J \Gamma_{d}(J) P_{g}+J \Gamma_{d}(-J) P_{c},
$$

which in turn is positive as the demon applies feedback on states $c$, as in Fig. 1(b). Consider $T_{s}=T_{d} \equiv T$. It can be shown that when $k_{B} T \tanh \left(J / 2 k_{B} T\right)<$ $(J / 4)\left(1+R_{d} / R_{s}\right)^{-1}$, Eq. (3) gives negative $\dot{Q}_{s}$, i.e., cooling, within a range of $0<|V|<\left|V_{\max }\right|<2 J / e$ (see Supplemental Material for derivation [32]). The entropy of the system then decreases as $\dot{S}_{s}=\dot{Q}_{s} / T<0$, seemingly against the second law; however, we still get $\dot{S}_{d}=$ $\dot{Q}_{d} / T \geq-\dot{Q}_{s} / T=\dot{S}_{s}, \quad$ resulting from Joule's law, $\dot{Q}_{s}+\dot{Q}_{d}=I V$.

Although energetically our device follows Joule's law, it is the information flow between the system and the demon that permits the decrease of system entropy. The mutual information between the system and the demon is 
$I_{m}=\ln \left(P_{n, N}\right)-\ln \left(P_{n}\right)-\ln \left(P_{N}\right)$, where $P_{n}$ and $P_{N}$ are the occupation probabilities of $n$ and $N$, respectively. As $P_{n=0}=P_{n=1}=P_{N=0}=P_{N=1}=0.5$, mutual information changes in a tunneling event from $g$ to $c$ as $\Delta I_{m, g \rightarrow c}=$ $\ln \left(P_{c} / P_{q}\right)$, and for $c \rightarrow g$ as $\Delta I_{m, c \rightarrow g}=-\Delta I_{m, g \rightarrow c}$ $[6,33,34]$. Tunneling events in the demon change mutual information at the rate

$$
\dot{I}_{m, d}=\ln \left(\frac{P_{c}}{P_{g}}\right) \Gamma_{d}(J) P_{g}+\ln \left(\frac{P_{g}}{P_{c}}\right) \Gamma_{d}(-J) P_{c} .
$$

The majority of the tunneling events in the demon are $c \rightarrow g$ transitions, and since $P_{g}>P_{c}, \dot{I}_{m, d}$ is positive. The rate of mutual information change by the system tunneling events is $\dot{I}_{m, s}=-\dot{I}_{m, d}$. As discussed in Ref. [34], the system heat generation satisfies $\dot{Q}_{s} \geq-k_{B} T_{s} \dot{I}_{m, d}$, implying that the maximum amount of cooling is bound by the amount of mutual information generated by the demon. Correspondingly, generating mutual information has a thermodynamic cost for the demon as $\dot{Q}_{d} \geq k_{B} T_{d} \dot{I}_{m, d}$. This can also be understood in terms of the configurational entropy $S_{\text {conf }}=-\ln [P(n, N)]$ as follows [34]: tunneling events in the demon bring the circuit from unlikely state $c$ to the more probable state $g$, decreasing $S_{\text {conf }}$. At least an equivalent of heat must be dissipated to satisfy the second law. On the other hand, most of the tunneling events in the system bring the setup to a more improbable state $c$, increasing configurational entropy. The second law then allows cooling by at most the amount of configurational entropy decreased; i.e., $-\Delta S_{s} \leq \Delta S_{\text {conf }}$. We note that in the limit $R_{d} \ll R_{s}, P(n, N)$ follows the thermal equilibrium distribution of the demon. Then $\ln \left(P_{g} / P_{c}\right)=J / k_{B} T_{d}$ such that $\dot{I}_{m, d}=\dot{Q}_{d} / k_{B} T_{d}$ by Eqs. (4) and (5). This implies that measurement of heat generated in the demon is also a direct measurement of information extracted by the demon.

Figure 2(a) shows a scanning electron micrograph of the experimental realization of Maxwell's demon. It was fabricated by standard electron beam lithography combined with shadow evaporation [35] of copper (normal metal) and aluminum (superconductor) metal films. Our device has the following parameters: $E_{s} / k_{B} \simeq 1.7 \mathrm{~K}, E_{d} / k_{B} \simeq 810 \mathrm{mK}$, $J / k_{B} \simeq 350 \mathrm{mK}, R_{s} \simeq 580 \mathrm{k} \Omega$, and $R_{d} \simeq 43 \mathrm{k} \Omega$ (two parallel junctions each with $\simeq 85 \mathrm{k} \Omega$ tunneling resistance). The fully normal system and demon junctions are realized with the laterally proximized aluminum dot technique [36]. We determine the heat generated in the left $(L)$ and right $(R)$ lead of the system as well as the lead of the demon by measuring the respective temperatures $T_{L}, T_{R}$, and $T_{d}$, as indicated in Fig. 2(a). This is achieved by reading the voltage of current-biased normal metalinsulator-superconductor junctions; see, e.g., Ref. [37]. Finally, the leads of the system and the demon are interrupted with direct contacts to superconducting leads, which permit charge transport by Andreev processes [38] but block heat transport at low temperatures. The structure is measured in a ${ }^{3} \mathrm{He} /{ }^{4} \mathrm{He}$ dilution refrigerator at the bath
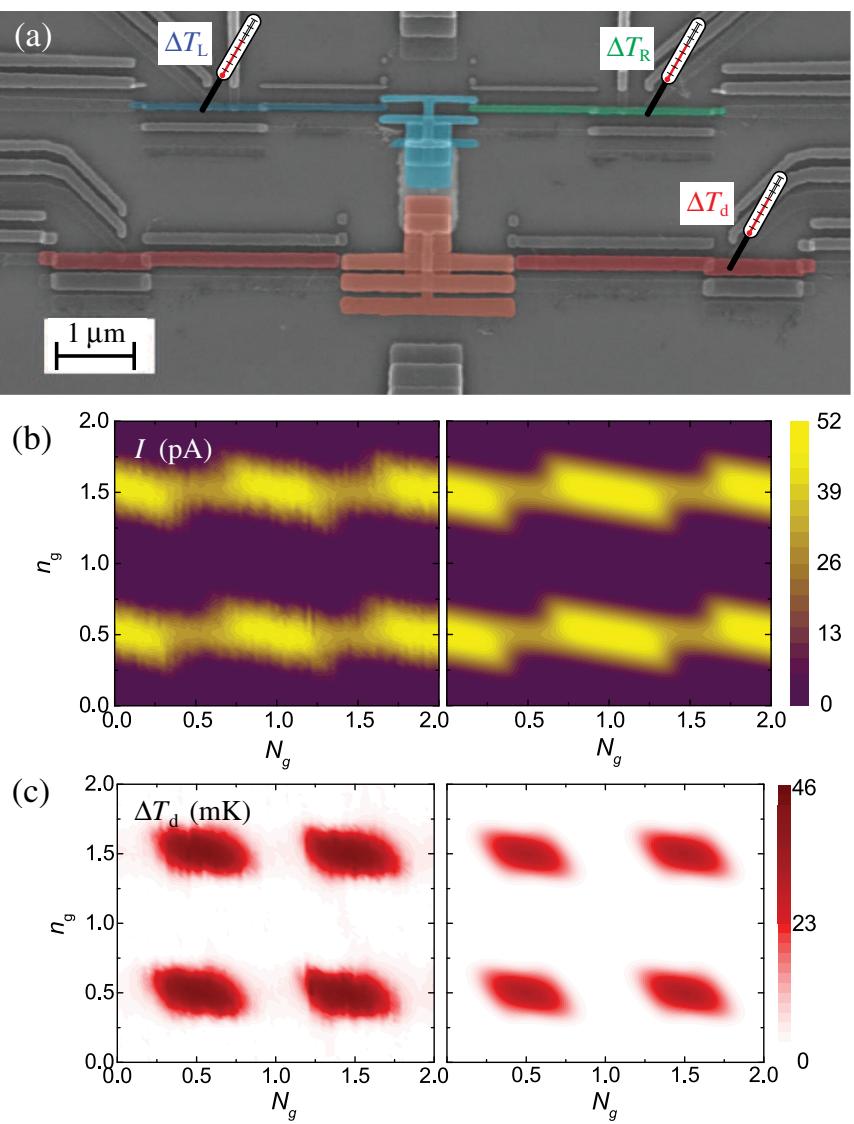

FIG. 2 (color online). Experimental realization. (a) A scanning electron micrograph of the structure. False color identifies the system island (light blue), its left lead (dark blue), and right lead (dark green), as well as the demon island (orange) and its leads (red). The system temperature deviations from their base value, $\Delta T_{L}, \Delta T_{R}$, and $\Delta T_{d}$, are measured at the indicated locations (see Supplemental Material for details of measurement setup [32]). (b) $I$ at $V=120 \mu \mathrm{V}$. When $N_{g}$ is an integer, $I$ is modulated by $n_{g}$ as in a standard SET. When $N_{g} \sim 0.5, I$ is smaller due to demon interaction. (c) $\Delta T_{d}$ at $V=120 \mu \mathrm{V}$. When $n_{g}, N_{g} \sim 0.5, \Delta T_{d}$ elevates due to the information flow between the system and the demon. Measured data in (b) and (c) are shown on the left and numerically obtained predictions on the right.

temperature of $40 \mathrm{mK}$. Details on the device fabrication and measurement configuration are given in the Supplemental Material [32].

The continuous heat generation is mediated primarily by lattice phonons that couple with the conduction electron heat bath at temperature $T_{L / R / d}$, contributing $\dot{Q}_{m, \mathrm{ph}}=$ $\Sigma \mathcal{V}_{m}\left(T_{0, m}^{5}-T_{m}^{5}\right), m=L, R$ or $d$, where $\Sigma$ is a materialspecific constant, $\mathcal{V}_{m}$ is the volume of the circuit element, and $T_{0, m}$ is the base temperature [39]. For the left and right electrodes of the system, $\mathcal{V}_{L / R} \approx 2.8 \mu \mathrm{m} \times 70 \mathrm{~nm} \times 20 \mathrm{~nm}$. Its island is approximately twice as large in volume. The demon has the total volume $\mathcal{V}_{d} \approx 4 \times 3.2 \mu \mathrm{m} \times 150 \mathrm{~nm} \times$ $20 \mathrm{~nm}$. We use $\Sigma \approx 4 \times 10^{9} \mathrm{~W} \mathrm{~m}^{-3} \mathrm{~K}^{-5}$ for $\mathrm{Cu}$. The rate of electron tunneling $\left(10^{6} \mathrm{~Hz}\right)$ in our device is faster than the phonon relaxation rate $\left(10^{4} \mathrm{~Hz}\right)$; however, it is small compared to the inelastic electron-electron relaxation rate, 
which is typically of the order of $10^{9} \mathrm{~Hz}$ [40], allowing the electrodes to equilibrate to an effective electron temperature $T_{m}$ that deviates from $T_{0, m}$. Furthermore, the temperature change caused by an individual tunneling electron is sufficiently small so that $Q_{m} / T_{m}$ is a good approximation for the entropy change. The temperature $T_{m}$ equilibrates such that the net heat generation is zero; i.e., $\dot{Q}_{m}=-\dot{Q}_{\mathrm{ph}, m}$. The base temperature $T_{0, m}$ is measured at $n_{g}=N_{g}=0$, where the state is Coulomb blockaded to $n=N=0$ corresponding to the energy minimum in Eq. (1) and no heat is generated in the circuit. Figure 2(b) shows that charge current $I$ in the system modulates with $n_{g}$ as in a standard SET. However, when $N_{g}=0.5$, the maximum measured current is smaller due to the feedback by the demon. Figure 2(c) demonstrates how at $n_{g}=N_{g}=0.5$ the heat generated in the demon is maximized for extracting information of the transported electrons.

The main result of this Letter is presented in Fig. 3(a), showing our observation at $V=20 \mu \mathrm{V} \simeq 2 \mathrm{~J} / 3 e$ of how the system cools down and its entropy decreases.
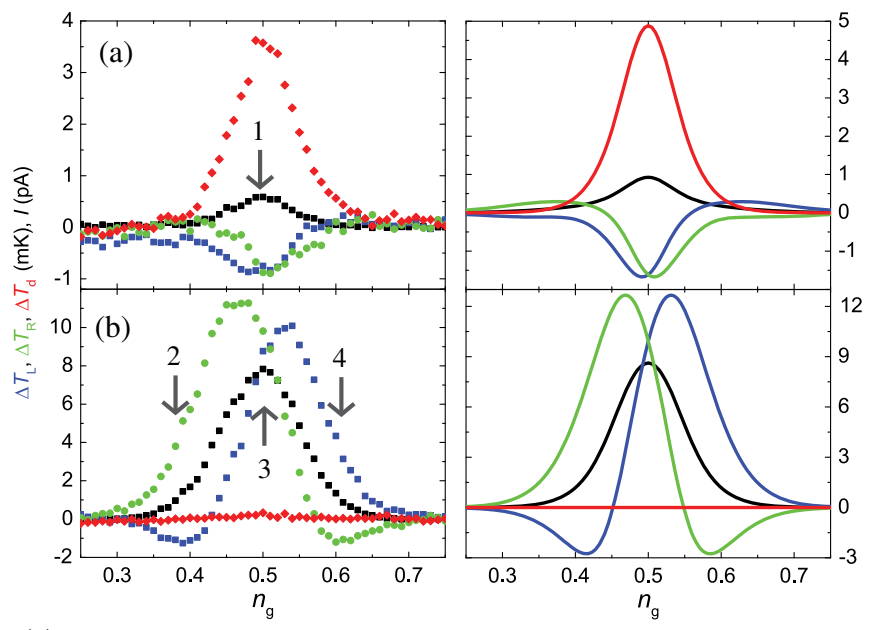

(c) 1
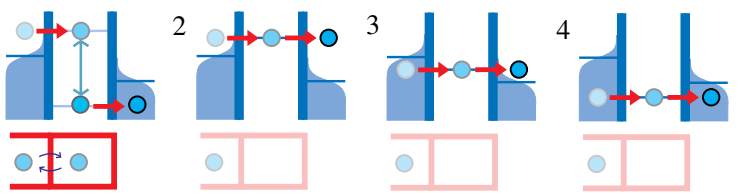

FIG. 3 (color online). Operation as a Maxwell's demon and as a one-sided refrigerator. Quantities shown are $I$ (black), $\Delta T_{L}$ (blue), $\Delta T_{R}$ (green), $\Delta T_{d}$ (red), with parameter values $V=20 \mu \mathrm{V}, T_{0, s}=77 \mathrm{mK}$, and $T_{0, d}=55 \mathrm{mK}$. (a) Measurement at $N_{g}=0.5$ (Maxwell's demon). Both $T_{L}$ and $T_{R}$ decrease, indicating overall cooling of the system. This is justified by the mutual information transfer between the system and the demon, which in turn generates heat in the demon, observed as elevated $T_{d}$. (b) Measurement at $N_{g}=0$ (SET refrigeration [41,42]). Either $\Delta T_{L}$ or $\Delta T_{R}$ can be negative, however, not simultaneously: overall heat is generated in the system. Measured data (symbols) are shown on the left and numerically obtained predictions (lines) on the right. (c) Energetics at different operation points, indicated as numbers in (a) and (b). At the operation point 1, the demon is interacting with the system as in Fig. 1(b). At operation points $2-4$, the demon is inactive.
Simultaneously, we observe how the demon, which collects the information and immediately applies a feedback to the system, generates heat as a necessary thermodynamic cost for extracting information from the system. On the other hand, Fig. 3(b) shows unchanged $T_{d}$ at $N_{g}=0$ since the demon is effectively uncoupled from the system as its state is locked to $N=0$. With that Coulomb blockade refrigeration $[41,42]$ occurs when $n_{g}$ deviates from 0.5 by causing either the left or the right lead to cool down, but overall heat is generated and entropy is produced in the system.

Figure 4(a) shows a measurement of current (inset) and temperatures as a function of $V$ at $n_{g}=N_{g}=0.5$. Increasing voltage bias boosts the rate of electrons passing through the system, however, at the cost of lower entropy decrease per electron. Furthermore, the risk of electrons to pass through the system without feedback control from the demon increases, in particular, via multielectron tunneling (see Supplemental Material for details [32]). Figure 4(b) compares the heat and mutual information produced by the demon, demonstrating that they differ by less than $15 \%$ for low $V$. The data shown in Fig. 4(c) show improvement of entropy decrease up to $20 \mu \mathrm{V}$, beyond which errors in the feedback process overcome the benefit of enhanced rate of
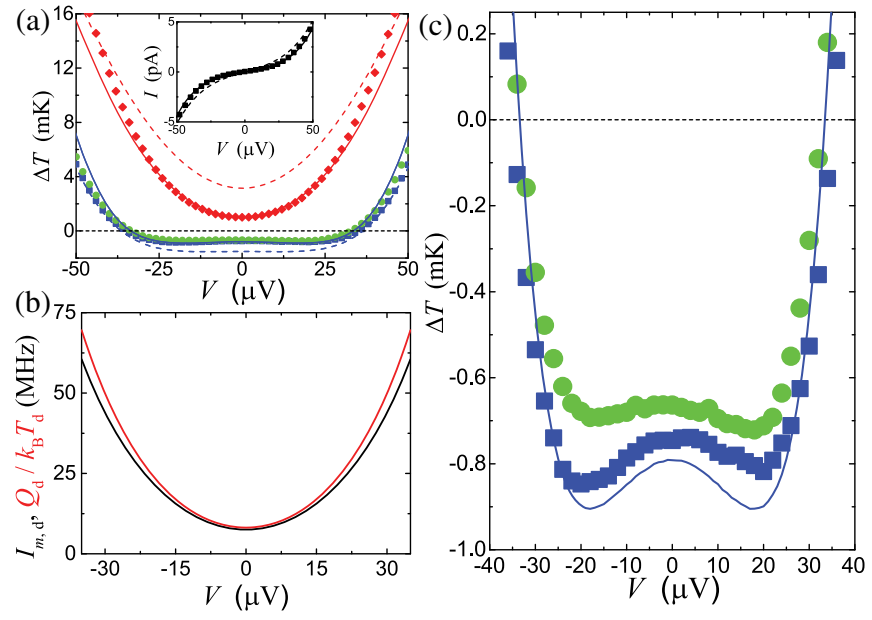

FIG. 4 (color online). Bias dependence. Here, $n_{g}=0.5$, $N_{g}=0.5$, and $T_{0, d}=55 \mathrm{mK}$. The data points (symbols) are obtained by averaging over 210 repetitions. (a) $\Delta T_{L}$ (blue squares), $\Delta T_{R}$ (green circles), and $\Delta T_{d}$ (red diamonds) with their respective prediction with $T_{0, s}=77 \mathrm{mK}$ (dashed lines) and $T_{0, s}=62 \mathrm{mK}$ (solid lines). Inset: $I$ in the same measurement. Applying voltage increases the number of electrons passing through the system and in turn the information flow between the system and the demon. This is observed as increased $T_{d}$. (b) Numerical comparison between $\dot{Q}_{d} / k_{B} T_{d}$ and $\dot{I}_{m, d}$, demonstrating that the two quantities match. (c) Enlarged view of the measured $\Delta T_{L}$ (blue squares) and $\Delta T_{R}$ (green circles). Increasing voltage bias further enhances the entropy decrease in the system up to about $\pm 20 \mu \mathrm{V}$. The model assumes a perfectly symmetric system and therefore predicts equal $\Delta T_{L}$ and $\Delta T_{R}$ with the fit $T_{0, s}=62 \mathrm{mK}$ (solid line). 
electron injection. At this voltage, the cooling power on the system is estimated to be $-\dot{Q}_{s} \approx 6 \mathrm{aW}$, while the heat dissipation in the demon is $\dot{Q}_{d} \approx 19 \mathrm{aW}$. Based on the heat generation, the mutual information production rate by the demon is then $\dot{I}_{m, d} \approx 25 \times 10^{6} \mathrm{~Hz}$. The current is $I \approx 600 \mathrm{fA}$; i.e., $\sim 4 \times 10^{6}$ electrons cross the system per second. Should successful feedback be performed for every electron, the heat generated by the demon would be $I \times 2 J / e \approx 36 \mathrm{aW}$. Experimentally, we observe $\approx 52 \%$ of this value; i.e., this fraction of the electrons transported through the system are successfully feedback controlled by the demon. For efficiency at maximal cooling power, $-\dot{Q}_{s} / I V$, we then get $\approx 0.56$.

In conclusion, we have realized and demonstrated experimentally a physically transparent autonomous Maxwell's demon on a chip, based on coupled singleelectron circuits undergoing tunneling events in a selfcontrolled manner. The demon acts on the system to decrease its entropy, observed as a temperature drop. The configuration allows one to measure the effect of the demon on the system, as well as to measure the thermodynamics of the demon itself. The device presented here demonstrates how information is transferred from the system to the demon, leading to heat generation in the demon in an amount that corresponds to the rate of information transfer. This setup constitutes a step towards autonomous information-powered nanodevices.

We thank Matthias Meschke, Antti Kemppinen, Joonas Peltonen, Felix Ritort, and Rafael Sánchez for useful discussions. We acknowledge financial support from the Academy of Finland Grants No. 272219 and No. 284594, the European Union Seventh Framework Programme INFERNOS (FP7/2007-2013) under Grant Agreement No. 308850, and the Väisälä Foundation. We acknowledge the availability of the facilities and technical support by Otaniemi research infrastructure for Micro and Nanotechnologies (OtaNano).

[1] Maxwell's Demon, edited by H. S. Leff and A. F. Rex (IOP Publishing, Bristol, 2003).

[2] L. Szilard, Z. Phys. 53, 840 (1929).

[3] R. Landauer, IBM J. Res. Dev. 5, 183 (1961).

[4] R. Landauer, Nature (London) 335, 779 (1988).

[5] T. Sagawa and M. Ueda, Phys. Rev. Lett. 100, 080403 (2008).

[6] T. Sagawa and M. Ueda, Phys. Rev. Lett. 104, 090602 (2010).

[7] D. Mandal and C. Jarzynski, Proc. Natl. Acad. Sci. U.S.A. 109, 11641 (2012).

[8] M. Esposito and G. Schaller, Europhys. Lett. 99, 30003 (2012).

[9] S. Deffner and C. Jarzynski, Phys. Rev. X 3, 041003 (2013).

[10] P. Strasberg, G. Schaller, T. Brandes, and C. Jarzynski, Phys. Rev. E 90, 062107 (2014).
[11] A. C. Barato and U. Seifert, Phys. Rev. Lett. 112, 090601 (2014).

[12] E. Lutz and S. Ciliberto, Phys. Today 68, 9, 30 (2015).

[13] V. Serreli, C.-F. Lee, E. R. Kay, and D. A. Leigh, Nature (London) 445, 523 (2007).

[14] G. N. Price, S. T. Bannerman, K. Viering, E. Narevicius, and M. G. Raizen, Phys. Rev. Lett. 100, 093004 (2008).

[15] J. J. Thorn, E. A. Schoene, T. Li, and D. A. Steck, Phys. Rev. Lett. 100, 240407 (2008).

[16] M. G. Raizen, Science 324, 1403 (2009).

[17] S. Toyabe, T. Sagawa, M. Ueda, E. Muneyuki, and M. Sano, Nat. Phys. 6, 988 (2010).

[18] A. Bérut, A. Arakelyan, A. Petrosyan, S. Ciliberto, R. Dillenschneider, and E. Lutz, Nature (London) 483, 187 (2011).

[19] E. Roldán, I. A. Martínez, J. M. R. Parrondo, and D. Petrov, Nat. Phys. 10, 457 (2014).

[20] J. V. Koski, V. F. Maisi, J. P. Pekola, and D. V. Averin, Proc. Natl. Acad. Sci. U.S.A. 111, 13786 (2014).

[21] J. V. Koski, V. F. Maisi, T. Sagawa, and J. P. Pekola, Phys. Rev. Lett. 113, 030601 (2014).

[22] J. M. R. Parrondo, J. M. Horowitz, and T. Sagawa, Nat. Phys. 11, 131 (2015).

[23] K. Chida, K. Nishiguchi, G. Yamahata, H. Tanaka, and A. Fujiwara, Appl. Phys. Lett. 107, 073110 (2015).

[24] A. C. Barato and U. Seifert, Europhys. Lett. 101, 60001 (2013).

[25] P. Strasberg, G. Schaller, T. Brandes, and M. Esposito, Phys. Rev. Lett. 110, 040601 (2013).

[26] N. Shirashi, S. Ito, K. Kawaguchi, and T. Sagawa, New J. Phys. 17, 045012 (2015).

[27] D. V. Averin and K. K. Likharev, J. Low Temp. Phys. 62, 345 (1986).

[28] R. Sánchez and M. Büttiker, Europhys. Lett. 100, 47008 (2012).

[29] Y. Zhang, G. Lin, and J. Chen, Phys. Rev. E 91, 052118 (2015).

[30] P. M. Solomon, P. J. Price, D. J. Frank, and D. C. La Tulipe, Phys. Rev. Lett. 63, 2508 (1989).

[31] D. V. Averin, A. N. Korotkov, and Yu. V. Nazarov, Phys. Rev. Lett. 66, 2818 (1991).

[32] See Supplemental Material at http://link.aps.org/ supplemental/10.1103/PhysRevLett.115.260602 for details about device fabrication and multielectron tunneling.

[33] D. Abreu and U. Seifert, Phys. Rev. Lett. 108, 030601 (2012).

[34] J. M. Horowitz and M. Esposito, Phys. Rev. X 4, 031015 (2014).

[35] G. J. Dolan, Appl. Phys. Lett. 31, 337 (1977).

[36] J. V. Koski, J. T. Peltonen, M. Meschke, and J. P. Pekola, Appl. Phys. Lett. 98, 203501 (2011).

[37] M. Nahum, T. M. Eiles, and J. M. Martinis, Appl. Phys. Lett. 65, 3123 (1994).

[38] A. F. Andreev, J. Exp. Theor. Phys. 19, 1228 (1964).

[39] F. C. Wellstood, C. Urbina, and J. Clarke, Phys. Rev. B 49, 5942 (1994).

[40] H. Pothier, S. Guéron, N. O. Birge, D. Esteve, and M. H. Devoret, Phys. Rev. Lett. 79, 3490 (1997).

[41] J. P. Pekola, J. V. Koski, and D. V. Averin, Phys. Rev. B 89, 081309(R) (2014).

[42] A. V. Feshchenko, J. V. Koski, and J. P. Pekola, Phys. Rev. B 90, 201407(R) (2014). 